\title{
Multiresolution Network Temporal and Spatial Scheduling Model of Scenic Spot
}

\author{
Peng Ge, Zhixue Liao, Chang Liu, Peiyu Ren, and Zhaoxia Guo
}

Business School, Sichuan University, Sichuan 610064, China

Correspondence should be addressed to Peiyu Ren; renpy.scu@163.com

Received 9 January 2013; Accepted 4 March 2013

Academic Editor: Yang Tang

Copyright (C) 2013 Peng Ge et al. This is an open access article distributed under the Creative Commons Attribution License, which permits unrestricted use, distribution, and reproduction in any medium, provided the original work is properly cited.

\begin{abstract}
Tourism is one of pillar industries of the world economy. Low-carbon tourism will be the mainstream direction of the scenic spots' development, and the $\omega$ path of low-carbon tourism development is to develop economy and protect environment simultaneously. However, as the tourists' quantity is increasing, the loads of scenic spots are out of control. And the instantaneous overload in some spots caused the image phenomenon of full capacity of the whole scenic spot. Therefore, realizing the real-time schedule becomes the primary purpose of scenic spot's management. This paper divides the tourism distribution system into several logically related subsystems and constructs a temporal and spatial multiresolution network scheduling model according to the regularity of scenic spots' overload phenomenon in time and space. It also defines dynamic distribution probability and equivalent dynamic demand to realize the real-time prediction. We define gravitational function between fields and takes it as the utility of schedule, after resolving the transportation model of each resolution, it achieves hierarchical balance between demand and capacity of the system. The last part of the paper analyzes the time complexity of constructing a multiresolution distribution system.
\end{abstract}

\section{Introduction}

Tourism is an emerging industry which can develop economy without damaging environment all around the world [1]. The development of tourism is not only the scenic ticket sales growth, but also to drive the development of other related industries [2]. When the rapid development of tourism gives a huge impetus to the economic development of the region, it also brings about a greater negative impact to the scenic ecological, social, and cultural environment. China's holiday system featured by "Golden Week" makes it easy to form a travel peak for the popular scenic spots, so that this contradiction is more prominent. After a long time of scenic field research, we found that the scenic overload is caused by tourists scale overload which can be divided into two categories: true overload and pseudo-overload. Distinguishing between them, true overload is that the scenic total temporal and spatial carrying load exceeds the capacity of scenic resources, which is decided by the structural handicaps of the scenic carrying capacity. In the case of not expanding the scenic scale, true overload must be completely resolved by limiting the travel scale and advocating rational travel.
However, pseudo-overload is caused by scenic structural contradictions under the situation that the number of tourists has not reached the real capacity of the scenic. Due to the lack of scientific control of tourists, some certain attraction nodes' tourists overload instantaneously caused local congestion and irreversible damage to the ecological environment of these regions and greatly reduced tourists' satisfaction. At present, most of the problem encountered belongs to the latter category; such pseudo-overload phenomenon has a certain amount of regularity on time and space. Therefore strengthening the management in scenic management at the peak of the tourism becomes the common focus of the scenic managers and scholars.

Domestic and foreign scholars have researched scenic sustainable development and its related aspects, such as scenic environment capacity [3, 4], management and services of tourism [5, 6], tourists' guide management [7], and scenic vehicle traffic [8-10]. The scenic shunt scheduling system is a complicated system which can be influenced by many factors. On the one hand it has to meet shunt need and to maximize tourists' satisfaction; on the other hand, because their activities' scope is in natural areas, it is necessary to 
consider the sustainable development of scenic area which needs to balance the load of all attraction nodes in the scenic and avoid traffic jams. Because of shunt needs and scheduling resources' limitedness, we cannot get both real time and optimality on the tourists shunt management. Since the 1990s in the field of statistical physics, complex network theory [11] thought that the behavioral characteristics of complex systems are not due to the complex dynamic behavior of a single attraction node but a large number of attraction nodes and their complex association mode. Ravasz et al. proposed a hierarchical module network model when they found that some network in reality has the feature of stratification and module [12]. Currently, the multi-resolution network model has been used to describe a variety of complex systems [13-15], and lots of research had involved network control [16-18], but there were few studies on the scenic shunt scheduling system. Zhang [19] studied scheduling decision system based on RFID smart card and divided the tourists' type based on the tourism path and then calculated the vehicle scheduling by the genetic algorithm program. But there is lots of redundant information in the actual system, which makes real-time scheduling scheme's global solving time consuming and unnecessary. Therefore, constructing scenic area network which can be changed with the state of the system will greatly enhance the efficiency of scenic shunt scheduling.

The time and space characteristics of the scenic shunt scheduling network accord with the time variability of the complex network structure systems; Therefore, Ren peiyu (2008) proposed the temporal and spatial shunt navigation management mode in National High Technology Research and Development Program (863 Program); he integrated information technology with the management techniques in scenic tourism engineering. In order to build a scenic management dissipative structure, he used a computer simulation scheduling engine to offer a basis for the scenic decision management for scenic managers, achieved the dynamic predictable scheduling of scenery and finally, alleviated the damage to the ecology during the tourism peak. On the basis of the established information systems, according to the timely forecast information feedback, developing a reasonable scenic scheduling program and then achieving scenic sustainable tourism development are the fundamental purpose of the scenic scheduling system research. This paper intends to build the scenic shunt multi-resolution network structure, collect data from bottom to up, forecast the shortterm demand of attraction nodes in scenery by the equivalent demand, and make the ability balance from top to down. This multi-resolution network structure provides a highly efficient platform for real-time shunt scheduling of scenery.

\section{Problem Analysis}

What should be solved in this paper is essentially a predictive scheduling problem. The principle is scheduling on the basis of the prediction, which can realize balanced distribution of the tourists at each attraction node in the scenery. At time $t$, it can be described by the following mathematical model:

$$
\begin{array}{ll}
\min & z(t)=\sum_{i=1}^{n} k_{i} y(t+1)_{i}^{2} \\
\text { s.t } \quad\left\{\begin{array}{l}
y(t+1)=(u(t) x(t+1)-I) \\
x(t+1)=f(x(t)) \\
w k_{i}>y(t+1)_{i} \\
w k_{i}>-y(t+1)_{i} \\
k_{i}=0 \text { or } 1,
\end{array}\right.
\end{array}
$$

where $y(t+1)=\left(y_{i}(t+1)\right)_{n \times 1}$ is a column vector, and $y_{i}(t+1)$ indicates the deviation between the real tourists numbers and the capacity of attraction node $i$ at time $t+1$; $x(t+1)=\left(x_{i}(t+1)\right)_{n \times 1}$ is an column vector, and $x_{i}(t+1)$ indicates the tourists number of attraction node $i$ at time $t+1 ; u(t)=\left(u_{i j}(t)\right)_{n \times n}$ is a $n \times n$ matrix, and $u_{i j}$ refers to the proportion of tourists on the attraction node $j$ which will arrive at attraction node $i$ next time at the time $t+1 ; I=\left(I_{i}\right)_{n \times 1}$ is a column vector, and $I_{i}$ refers to the capacity of attraction node $i ; f(x)$ is a function used to forecast the tourists on all attraction nodes at time $t+1$ based on the tourists data at the item $t ; w$ is an infinite number.

\section{Building Scenic Multi-Resolution Network}

Resolution refers to the detail level of a model to describe the real world; it is decided by the detail level of cognitive levels, attributes, input, output, time, process, and so on. The multi-resolution network involves in multiple levels and many aspects, including cognitive level static characteristics (state properties, input parameters, output parameters, law) and dynamic characteristics (time and process) [20].

As the present problem is becoming increasingly complex, and the scale is expanding quickly, establishing different resolution models and getting these models together to study are the inevitable trend of modeling complex systems according to the different needs of the problem. Also observing things and considering the problem from different resolution are an important feature of the human mind. Therefore, multi-resolution modeling gradually becomes a hot research field of modeling and simulation in the international area since the 1990s [21]. Caughlin and Sisti [22] understood multi-resolution modeling problems from the abstract aspect.

Multi-resolution modeling is mainly used in the simulation of complex systems, such as combat simulation [23]. Because the scenery is a complex open giant system through that people can interact with nature, the elements are numerous, the relationship is complex, and the target function is versatile. It is a complex spatial and temporal structure, which presents characteristics of holistic, dynamic, nonlinear, self-adaptive, and multidimensional. Building scenic multiresolution models has practical significance, but very few scholars have done this research. This paper will build the scenic temporal and spatial scheduling multi-resolution network, which will be taken as the basis of the scenic temporal and spatial scheduling. 


\subsection{The Definition of Scenic Multi-Resolution Network}

Definition 1. The attraction modes with the capable of independent operation which are divided by the grid (e.g., $I_{1}-$ $I_{311}$ in Figure 1) is called field, which can represent not only a single attraction node but also multiple attraction nodes which can satisfy certain conditions set.

Taking Figure 1, for example, the upstream domain of $I$ is marked $O I_{I}$, its subfield is marked $S I_{I}, I_{22}$ is upstream $I_{22}$, and $\left\{I_{21}, I_{22}, I_{23}\right\}$ is called the subfield set. In Particular, if $I$ is located in the lowest resolution layer, $O I$ is null; if $I$ is located in the highest-resolution layer, SI is null.

Definition 2. The minimum distance between two certain fields is called grid distance (reachable matrix, named $d$, of which unit is grid, called distance).

Taking Figure 2(b), for example, the distance from field $A$ to $E$ can be calculated as follows:

$$
d_{A E}=\min \left(d_{A B}+d_{B C}+d_{C D}+d_{D E}, d_{A D}+d_{D E}\right)=7
$$

Definition 3. The process of some fields with a certain distance merging into a larger field is called gathering.

Taking Figure 2(b), for example, the distance between fields $C$ and $D$ is 1 ; by the process of gathering, we can get a lager field $F$, and the distance from field $B$ to $F$ can be calculated as follows:

$$
d_{B F}=\frac{1}{2}\left(d_{B C}+d_{B D}\right)
$$

Definition 4. The field collection formed by fields on the different resolution by constantly gathering is called multiresolution layer.

The higher the resolution layer, the more visible the details. As shown in Figure 1, the resolution of the layers $L_{1}, L_{2}, L_{3}$ increases gradually. The high-resolution system shows more discrete dynamic characteristics, while the lowresolution layer shows a more steady-state characteristics.

\subsection{Scenic Temporal and Spatial Scheduling and Its Operation} Processes. Scenic multi-resolution network system is a layerby-layer recursive nested structure (see Figure 1), scenic's operation with spatial and temporal characteristics must be on the multi-resolution networe system above. Figure 3 shows the scenic temporal and spatial scheduling operation flow structure.

The engine functions are described as follows.

Prediction Engine. Predicition engine botton-up forecasts each field's equivalent demand at $\Delta t$ time later based on the system state and shunt probability at time $t$ and statistics these resources disributed in the field.

Matching Engine. Matching engine work from top to down, it is responsible for the match balance of the system requirements and capabilities; the same layer field determines the balance scheduling program by the demand balance model, layer by layer to activate and to build the specific scheduling program of the entire system.

Execution Engine. Execution engine performs scheduling scheme and generates the system state of time $t+\Delta t$, which will be taken as the input of the prediction engine next cycle.

\section{Demand Prediction Engine}

4.1. Demand Information Collection and Analysis. The tourist's RFID card can be collected by the RFID reader when he got in or out the attraction nodes in the scenery, so at time $t$ we can know the tourist number of each attraction node before time $t$ and which attraction nodes the tourists had visited. The detailed processes are showed in Figure 4.

4.2. Equivalent Demand. Actual system can gain scheduling needs and resource capacity information by the related equipment. However, due to the certain distance between the resources and needs grid, there must be a delay. Further, due to the change of external environment and the presence of measuring error, the prediction values are very random, which will cause a huge deviation of scheduling effect. To solve this problem, this paper introduces the equivalent demand, considering the impact of the upstream field on the target field of the dynamic shunt probability and delay during the forecast period.

Definition 5. Set that target field sends scheduling demand instruction at $t$ time, and the demand of the scheduling can be met at $t+\Delta t$ time, thus all the upstream field that is away from the target field within $\Delta t$ meters may affect the target field scheduling demand. Therefore we need to put these fields' current demand converted into target field scheduling demand, which is called equivalent demand (recorded as ER $(t \mid \Delta t))$.

Definition 6. The amount of resources of the target field at $t$ time is called resource capacity (recorded as CR $(t)$ ).

Considering the scenic state of a travel period, the demand at the same attraction nodes are significantly different at different time. There are also obvious differences among different attraction nodes at the same time, which reflects the dynamic process of the attraction nodes' demands in the temporal and spatial dimension.

There are two commonly used methods in forecasting the demands of field $j$; one considers all the cumulative impact of upstream field of field $j$; the other only considers the impact of the immediate vicinity upstream fields of field $j$. It essentially determines the prediction step $\Delta t$; the longer the step size, the greater the range of prediction time and the more fluctuations of prediction results. In contrast, if the step size is set too small, the convergence speed is too slow; the two situations all are unreasonable. In fact, the prediction step has a great influence on the prediction. The demand's forecast value of the target field at time $t+\Delta t$ is related to the current demand of the upstream fields which were transferred to the target fields. The affection factors are mainly shown in 


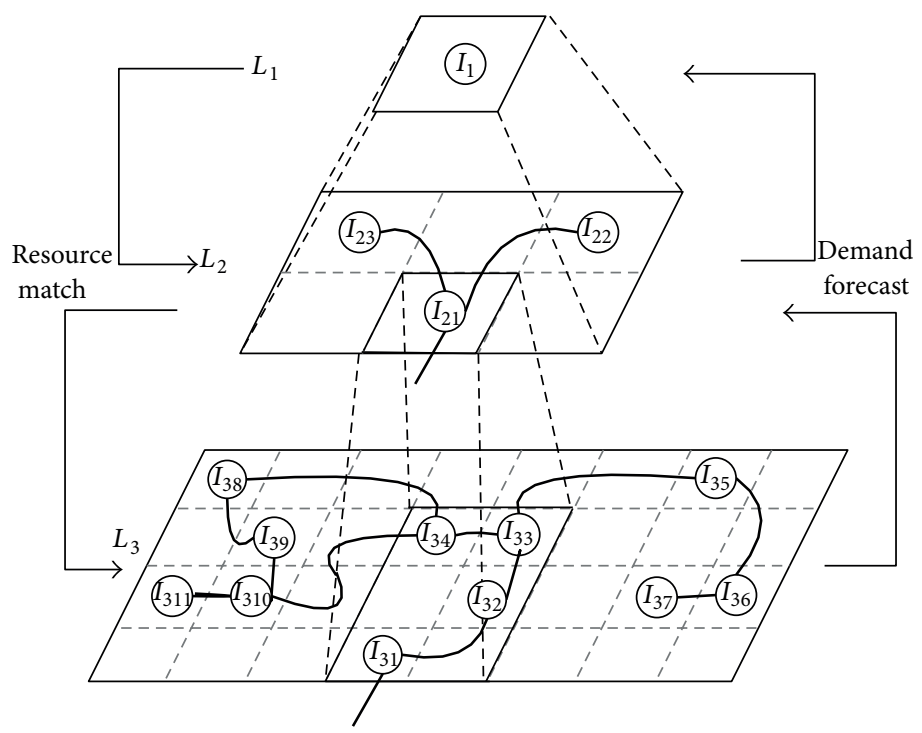

FIGURE 1: Multiresolution network system.

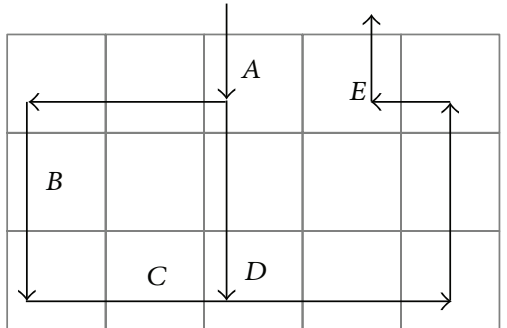

(a)

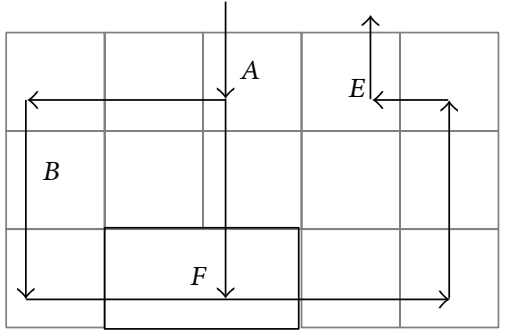

(b)

Figure 2: Grid distance.

two degrees: one is the shunt probability of upstream field to target field at time $t$, the other is the time distance $\left(t_{d}\right)$ between them. Considering the degree of the target field's demand influenced by the upper field may become serious or weak as the time distance changes. So we can use the time distance function $\omega\left(t_{d}\right)$ to describe the contribution degree of the field whose time distance is $t$ to the target field prediction. So $\omega^{\prime}\left(t_{d}\right)<0$, where $\omega^{\prime}\left(t_{d}\right)$ is the first derivative of $\omega\left(t_{d}\right)$. Figure 5 shows some possible images of function $\omega\left(t_{d}\right)$; the specific function will be calculated by the analysis of a large number of actual data. In this paper, we choose the linear function for simple operation. Set distance influence factor as $\varepsilon$; when upstream field's impact to target field is less then

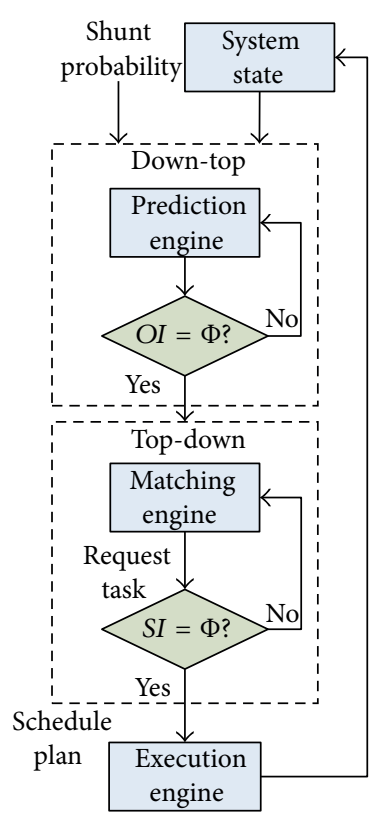

FIGURE 3: Process structure of multiresolution network temporal and spatial scheduling's operation.

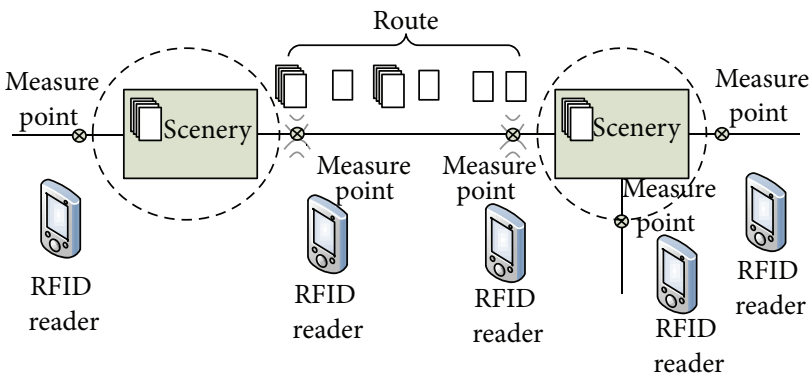

FIGURE 4: Demand information collection. 


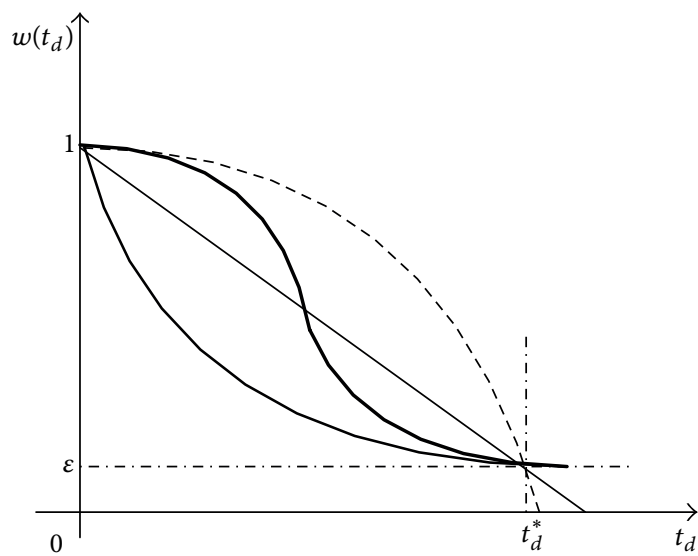

FIgURE 5: Function $w\left(t_{d}\right)$.

$\varepsilon$, we can neglect it; the time distance of $\omega\left(t_{d}\right)=\varepsilon$ is marked $t_{d}^{*}$, so the equivalent demand forecast can be finished by two steps; firstly, determine the maximum time distance which satisfies $w\left(t_{d}\right) \geq \varepsilon$, set forecast step size as $\Delta t$, and update $\Delta t=\min \left(t_{d}^{*}, \Delta t^{\prime}\right)$. Secondly, consider all fields within the time distance $\Delta t$ from target field, and change the demand at time $t$ to the target field's equivalent demand which is regarded as evidence of scheduling.

According to the above analysis, when $S I=\Phi$ (all fields have been located in the highest-resolution layer) equivalent demand of field $j$ can be expressed as follows:

$$
\begin{array}{r}
\mathrm{ER}_{j}(t \mid \Delta t)=N_{j}(t)+\sum_{i} p_{i j}(t) . \times N_{i}(t) . \times w\left(t_{i j}\right) \\
\left(i \in I, t_{i j} \leq \Delta t\right) .
\end{array}
$$

When $S I \neq \Phi$, forecast demand of field $j$ at time $t$ is the equivalent of each subfield's upgathering.

$$
\mathrm{ER}_{j}(t \mid \Delta t)=\sum_{i} \mathrm{ER}_{i}(t \mid \Delta t) \quad\left(i \in S I_{j}\right) .
$$

Similarly, ability of field $j$ at time $t$ is the equivalent of each subfield's upgathering.

$$
\mathrm{CR}_{j}(t)=\sum_{i} \mathrm{CR}_{i}(t) \quad\left(i \in S I_{j}\right) .
$$

\section{Demand Matching Engine}

The state of the attraction nodes in the scenery changes with the tourism activities. Therefore, building a multi-resolution network according to the system state and building a rapid equilibrium layers load algorithm model to improve the system's fast response ability is necessary. The approach ued in the previous studies on the simple systems or comples engineering systems is to take mathematics as a tool to establish the mathematical model and make system analysis. Inspired by system dynamics attractor, this paper intends to build Demand hierarchical balance matching model, which effectively controls the size of the entire system solving. The detailed Demand matching process is showed in Figure 6.

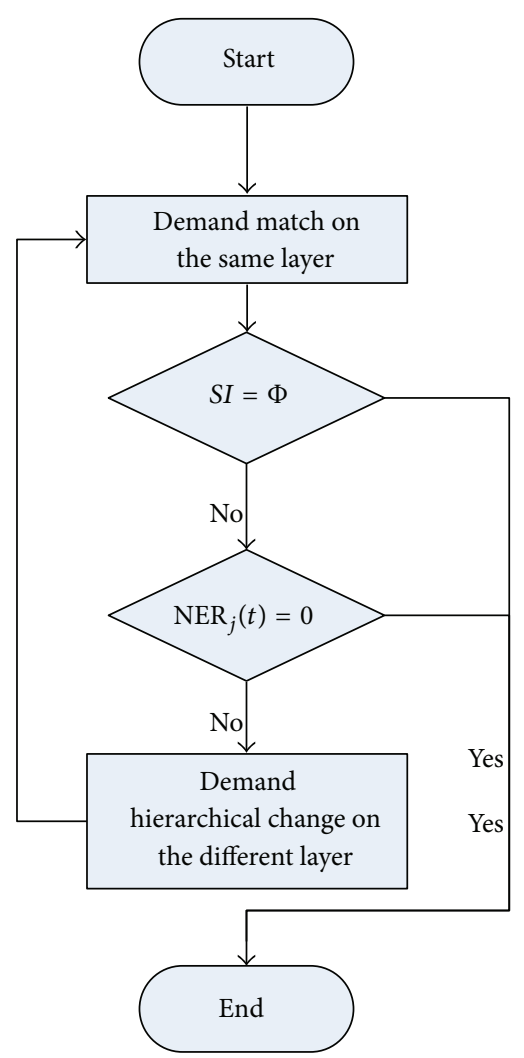

FIGURE 6: Demand matching flow chart.

5.1. Definition Demand-Capacity Hierarchical Balance Model. Demand balance is essential to balance the load, and it is an important means to achieve the effective sharing of resources. Dynamic load balancing must be established based on the real-time prediction of the state information of each fields. Therefore, real-time response is essential. The structure of the system at this time is of great influence on the complexity in calculating. In fact, even in the most simple case, the optimization of multidemand balance scheduling is an NP problem [24]. Therefore, the study often builds optimization algorithm based on a series of assumptions. For computing resources, Yagoubi and Slimani found a loadbalancing algorithm based on the hierarchy, which represents the load network by the four tree structures [25]. Rashedi et al. [26] put forward the gravitation search algorithm (GSA), the optimization search technology, which is derived from the gravitation simulation in physics and is similar to the particle swarm optimization (PSO). In fact, the same layer field's Demand match problem is the classic transportation problem. This paper uses gravitation search algorithm to find the gravitation between the fields, which will be taken as a scheduling utility of the transportation problem.

GSA based on the law of gravitation thinks that there is gravitation between any two objects, which makes the object move forward to the larger mass object. In the same layer resolution, due to the imbalanced distribution of demand and capacity, some fields' equivalent demand is greater than the resources; however, some other fields are just the reverse and 
the gravitational force is generated between these two types of fields, which can let the ability in the resource field move to the demand field.

Definition 7. The difference between equivalent demand and resource ability of the field is called net demand (named NER), reacting the absolute state at the certain time. follows:

So, the net demand of field $j$ at time $t$ can be calculated as

$$
\mathrm{NER}_{j}(t)=\mathrm{ER}_{j}(t \mid \Delta t)-\mathrm{CR}_{j}(t) .
$$

Definition 8. The field of which the net demand is negative is called resource field, and resource field set is named RA.

Definition 9. The field of which the net demand is positive is called demand field, and demand field set is named EA.

Definition 10. The field of which the net demand is zero is called neural field, and neutral field set is named BA.

\subsection{Demand-Capacity Hierarchical Balance Model of the Same Layer}

Definition 11. The attraction node which needs resource in scenery is demand node.

Definition 12 (resource node). The attraction node which provides resource in scenery is called resource node.

Demand and capacity are two of the most critical elements in the scheduling process. How to match the demand and the ability is one of the goal of the scheduling process.

Normally, the greater the number of waiting tourists, the larger demand of resources. For resource nodes, the amount of resources is the number of the car.

The gravitational force is generated between the demand field and resource field, which is not only affected by the grid distance and direction, but also the net demand of the two fields. Grid distance and direction are determinded only by the relative position between the demand field and resource field. And demand and capacity depend on the state of the field in the current scheduling cycle. Use triples $F_{i j}(t)=$ $\left\{\left\langle d_{i j}, \operatorname{NER}_{i}(t / \Delta t), \operatorname{NER}_{j}(t / \Delta t)\right\rangle \mid i \in \mathrm{EA}, j \in \mathrm{RA}\right\}$ to show the attraction between demand field $i$ and resources field $j$. The gravitation standard formula is $F=G m M / r^{2}$. $G$ is the gravitational constant, $m$ and $M$ represent the quality of the two particles, respectively, and $r$ is the distance between the two particles.

Make corresponding conversion according to the research questions, set gravitational force as proportional to net demand of the two fields which generate gravitation and inversely proportional to square of the distance between the field, and gravitation constant is set as $r$. So, at time $t$ the two field's Demand matching gravitation is

$$
F_{i j}(t)=r \cdot \operatorname{NER}_{i}\left(\frac{t}{\Delta t}\right) \cdot \frac{\left|\operatorname{NER}_{j}(t / \Delta t)\right|}{d_{i j}^{2}} \quad(i \in \mathrm{EA}, j \in \mathrm{RA}) .
$$

We sort the gravitation which has been seen as scheduling effectiveness by its force, and distribute the schedule resource for the demand ordinally.

\section{The Analysis of Time Complexity in Scenic Spots' Dynamic Multi-Resolution System Building}

For the huge data information of shunting system in scenic spots, the solution of the traditional diversion scheduling model is time consuming. And it cannot adapt to the needs of real-time shunting. The scenic dynamic shunting network based on multi-resolution which this paper adopts views demand node as its center, by continually solving the Demand match gravity model, building the scenic shunt network layer by layer, and providing a platform for efficiently handling a large number of dynamic information. Under different circumstances, the differences of multi-resolution complexity are quite large, mainly affected by the number of demand field and the number of resolution layers at the same moment. Therefore the discussion of time complexity of building scenic multi-resolution shunt network is required to be unfolded step by step. The calculation of each layer algorithm time complexity can be divided into three parts. (1) Calculate each attraction node's shunt probability matrix $p_{i j}$ of prediction steps in the highest-resolution layer and calculate each attraction node's equivalent demand of the layer; the field is divided into three sets: neutralization field, demand field, and resource field. Assuming that the highest-resolution layer has $m$ attraction nodes, then the time complexity of calculating each attraction nodes' shunt probability matrix is $O\left(\mathrm{~m}^{2}\right)$; the required time complexity of calculating each attraction node's equivalent demand and divided collection is $O(m)$. (2) Respectively take demand field as the center, put the resource field within a certain distance of grid together, constitute the higher-resolution layer, take the highest-resolution layer as an example, and assume the three sets equally split every attraction node. The worst time complexity which the layer gathers is $O\left(m^{2}\right)$. (3) Parallelly calculate gravitational matrix $F_{i j}$ of each demand field and resource field layer by layer. Match Demand according to the order of gravitational force and increase sorting time complexity on the basis of step 2, marked as $O\left(m^{2}\right) * O(m *$ $\left.\log _{m}\right)=O\left(m^{3}\right)$. These three parts are sequence relationship, so the time complexity of the entire system building is $O\left(\mathrm{~m}^{2}\right)$. In fact, the calculations of step 2 and step 3 are parallel, so the time complexity is $O(m)$, and because the collection is divided, the quantity of every layer's actual demand field is much smaller than $m$. In the actual system, this will greatly shorten calculating time.

In order to clearly illustrate the effectiveness of the calculation ability of the model, we take the multi-resolution network system in Figure 1 as an example. As $m=11$, take $\log _{11} \approx 1$; for respective $m$ that is less than 11 , take $\log _{m} \approx 1$; the computation of its building process time frequency is shown in Table 1.

If we use the general task allocation planning model to solve the problem, time complexity is $O\left(\mathrm{~m}^{m}\right)$. In this 
TABLE 1: Algorithm time frequency.

\begin{tabular}{lcccc}
\hline Layer of resolution & $p_{i j}$ & Set division & Gathering & Demand-capacity match \\
\hline$k=1$ & $11^{2}$ & 11 & $3+3+2$ & $3 * 3+3 * 3+2 * 2$ \\
$k=2$ & 0 & 3 & 2 & 2 \\
$k=3$ & 0 & 1 & 0 & 0 \\
\hline Total & & & 170 \\
\hline
\end{tabular}

case, the time frequency is $(11-3)^{3}=512$. In this paper it is apparently more efficient to use the shunt scheduling model based on the multi-resolution network to calculate. In this paper, the scheduling model's calculation process in the highest-resolution layer is more complex, which determines the entire system's algorithm time complexity. However, for nonhighest-resolution layer, the fields' number of each layer after the gathering greatly is less than that of the previous layer, so it greatly decreases time frequency. Therefore, the complexity of the algorithm is mainly determined by the highest-resolution layer. Based on the above analysis of algorithm time frequency, it can be inferred that when faced with the large shunt demand of the scenic shunting system, and shunt resource capacity dispersed in different fields (i.e., the number of capacity field is large but the ability of each capacity field to provide is small), the scheduling model based on multi-resolution network has obvious advantages in computational efficiency. Also it can take full advantage of piecemeal residual shunting ability of the scenic system's shunt resources, improve the resources' utilization of the entire scenery, and provide an efficient platform.

\section{Conclusion}

In this paper, we use dynamic multi-resolution network to describe the temporal and spatial schedule system, construct the real-time prediction system and hierarchical balance model, and then give the detailed steps to solve the problem. The sixth section of this paper analyzes the time complexity in constructing the temporal and spatial scheduling multiresolution system and takes Figure 1 as an example to calculate the time frequency in constructing the multi-resolution network, showing that the top-down real-time prediction model and the bottom-up Demand hierarchical balance model based on multi-resolution structure which change the dynamic programming problems into multiple parallel linear assignment problem greatly simplify the problem's solving difficulty.

To make the temporal and spatial scheduling multiresolution network successfully implemented in the scenery, in addition to ensure the accuracy of scenic real-time status data, the effectiveness of the two parameters (the dynamic shunt probability function and distance effect function) in the equivalent demand forecast also plays a decisive role. The latter part of the study focuses on fitting dynamic shunt probability based on statistical data and analyzes the impact of different $w_{i j}(t)$ on system performance.

In addition, this paper does not consider the control of vehicle empty rate in the calculation of gravitation, which will affect the scenic scheduling carbon emissions to some extent. So further research will also focus on the analysis of the elements in gravity model.

\section{Acknowledgments}

This work was supported by the Major International Joint Research Programme of the National Natural Science Foundation of China (Grant no. 71020107027), the National High Technology Research and Development Major Programme of China (863 Programme) (Grant no. 2008AA04A107), the National Natural Science Foundation of China (Grant no. 71001075), the China Postdoctoral Science Foundation (Grant no. 2012M521704), 985, 211 projects of Sichuan University, and Central University Fund of Sichuan University.

\section{References}

[1] K. Lindberg, S. McCool, and G. Stankey, "Rethinking carrying capacity," Annals of Tourism Research, vol. 24, no. 2, pp. 461-465, 1997.

[2] A. Leask, A. Fyall, and P. Goulding, "Revenue management in Scottish visitor attractions," Yield Management: Strategies for the Service Industries, vol. 19, pp. 211-232, 2003.

[3] C. Z. Wu, "Huangshang scenic;s tourism environmental capacity current situation and control," Areal Research and Development, vol. 9, pp. 57-61, 1993.

[4] J. Guo and S. F. Zhang, "The preliminary analysis and regulation on tourism environment capacity in Eastern Suburb scenic area of Nanjing City," Journal of Nanjing Normal University (Natural Science Edition), vol. 26, pp. 120-124, 2003.

[5] S. F. McCool, "Planning for sustainable nature-dependent tourism development: the limits of acceptable change system," Tourism Recreation Research, vol. 19, no. 2, pp. 51-55, 1994.

[6] E. J. Pilcher, P. Newman, and R. E. Manning, "Understanding and managing experiential aspects of soundscapes at muir woods national monument," Environmental Management, vol. 43, no. 3, pp. 425-435, 2009.

[7] Y. P. Tseng, G. T. Kyle, C. S. Shafer, A. R. Graefe, T. A. Bradle, and M. A. Schuett, "Exploring the crowding-satisfaction relationship in recreational boating," Environmental Management, vol. 43, no. 3, pp. 496-507, 2009.

[8] Y. Shiftan, D. Vary, and D. Geyer, "Demand for park shuttle services-a stated-preference approach," Journal of Transport Geography, vol. 14, no. 1, pp. 52-59, 2006.

[9] S. Lawson, P. Newman, J. Choi, D. Pettebone, and B. Meldrum, "Integrated transportation and user capacity research in Yosemite National Park: the numbers game," Transportation Research Record, no. 2119, pp. 83-91, 2009. 
[10] L. Wang and C. Zhang, "Research on the frame of Intelligent Tourism Transportation System (ITTS)," Communications Standardization, vol. 11, pp. 150-153, 2005.

[11] M. T. Gastner and M. E. J. Newman, "Optimal design of spatial distribution networks," Physical Review E, vol. 74, no. 1, Article ID 016117, 2006.

[12] E. Ravasz, A. L. Somera, D. A. Mongru, Z. N. Oltvai, and A. L. Barabási, "Hierarchical organization of modularity in metabolic networks," Science, vol. 297, no. 5586, pp. 1551-1555, 2002.

[13] Y. Kong, "Research of optimal solution on robust graph coloring problem," Computer Knowledge and Technology, vol. 5, pp. 5773-5775, 2009.

[14] Y. X. Liu, C. Z. Han, and R. H. Li, "Event driven state space model for DEDS," Jiejiang University, vol. 8, pp. 193-197, 1983.

[15] Y. J. Lu and H. P. Dai, "Hybrid Petri net modeling for urban traffic network," Journal of Zhejiang University(Engineering Science), vol. 41, no. 6, pp. 930-934, 2007.

[16] Y. Tang, Z. D. Wang, H. J. Gao et al., "A constrained evolutionary computation method for detecting controlling regions of cortical networks," IEEE/ACM Transactions on Computational Biology and Bioinformatics, vol. 9, no. 6, pp. 1569-1581, 2012.

[17] Y. Tang and W. K. Wong, "Distributed synchronization of coupled neural networks via randomly occurring control," IEEE Transactions on Neural Networks and Learning Systems, vol. 24, no. 3, pp. 435-447, 2013.

[18] E. Tian and D. Yue, "A new state feedback $H_{\infty}$ control of networked control systems with time-varying network conditions," Journal of the Franklin Institute, vol. 349, no. 3, pp. 891-914, 2012.

[19] X. L. Zhang, "On accurate visitor management in scenic spots based on RFID technique," Scientific and Technological Management of Land and Resources, vol. 8, pp. 89-93, 2009.

[20] Y. B. Du, J. J. Yang, W. Lu, and T. L. Niu, "Study of relevant conceptions about multi-resolution modeling and simulation," Journal of System Simulation, vol. 20, no. 6, pp. 1386-1389, 2008.

[21] B. H. Liu and K. D. Huang, "Multi-resolution modeling: present status and trend," Acta Simulata Systematica Sinica, vol. 16, pp. 1150-1154, 2004.

[22] D. Caughlin and A. F. Sisti, "A summary of model abstraction techniques," in Enabling Technology for Simulation Science, vol. 3083 of Proceeding of SPIE, 1998.

[23] M. Z. Li, C. J. Bi, and X. B. Guo, "A research on air force combat simulation modeling based on multi-resolution," Command Control and Simulation, vol. 1, pp. 51-55, 2008.

[24] M. R. Garey and D. S. Johnson, “'Strong” NP-completeness results: motivation, examples, and implications," Journal of the Association for Computing Machinery, vol. 25, no. 3, pp. 499$508,1978$.

[25] B. Yagoubi and Y. Slimani, "Dynamic load balancing strategy for grid computing," Transactions on Engineering Computing and Technology, vol. 13, pp. 1305-5313, 2006.

[26] E. Rashedi, H. Nezamabadi-Pour, and S. Saryazdi, "GSA: a gravitational search algorithm," Information Sciences, vol. 179, no. 13, pp. 2232-2248, 2009. 


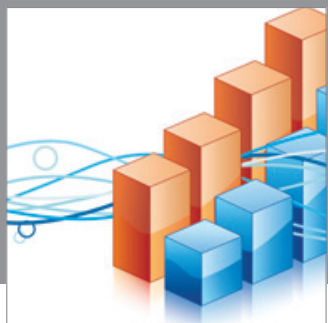

Advances in

Operations Research

mansans

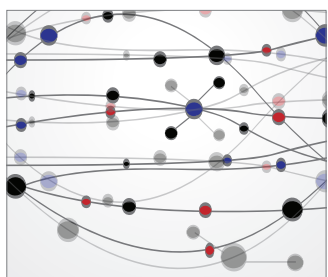

The Scientific World Journal
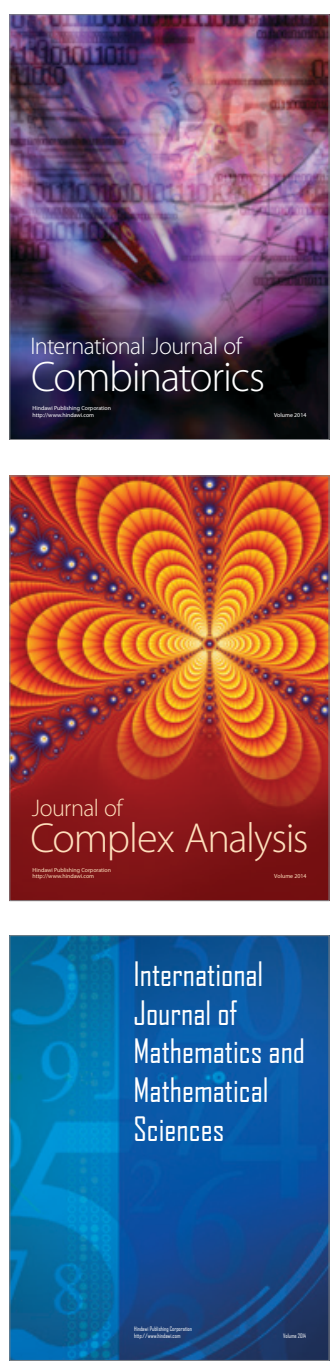
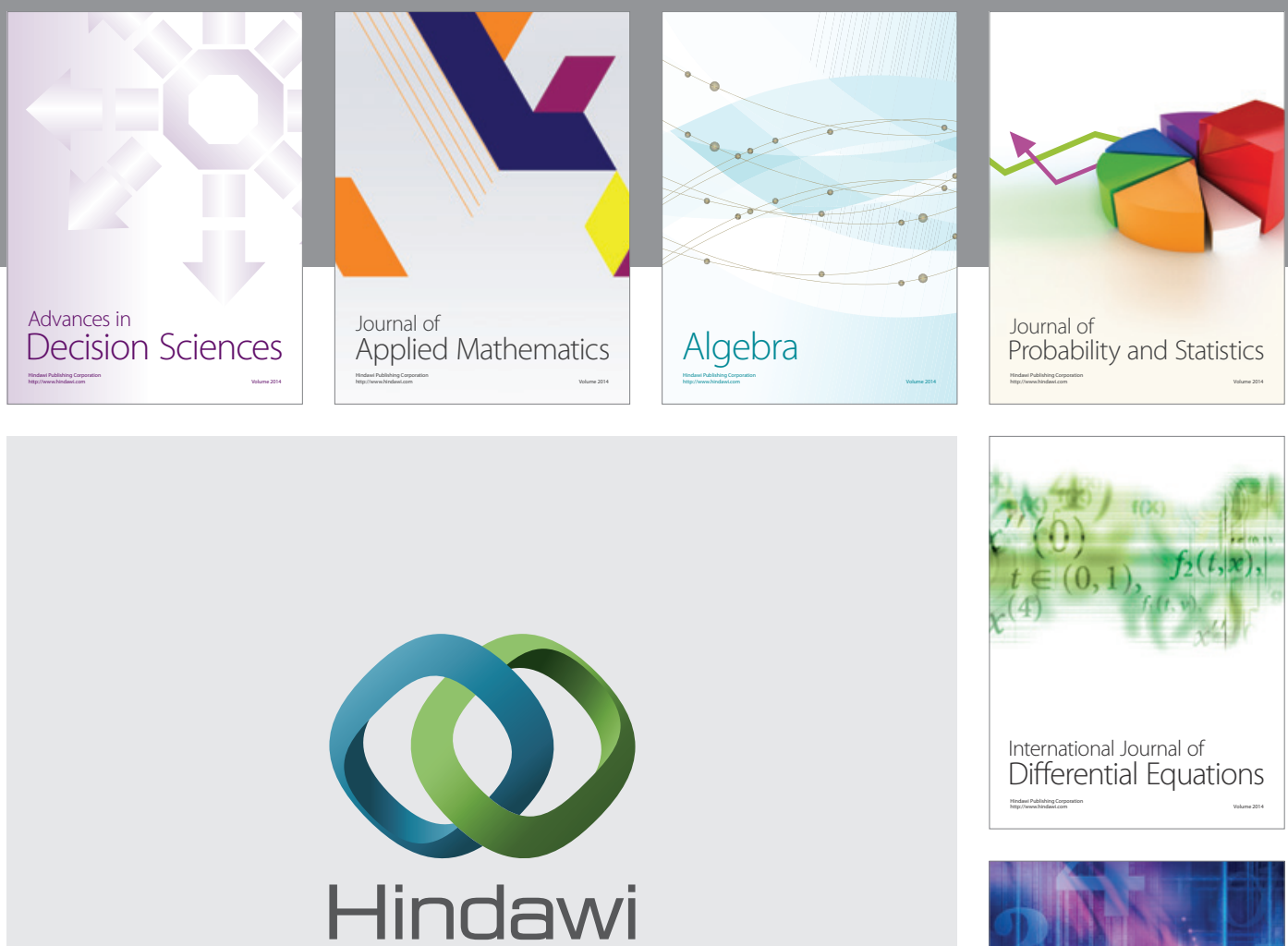

Submit your manuscripts at http://www.hindawi.com
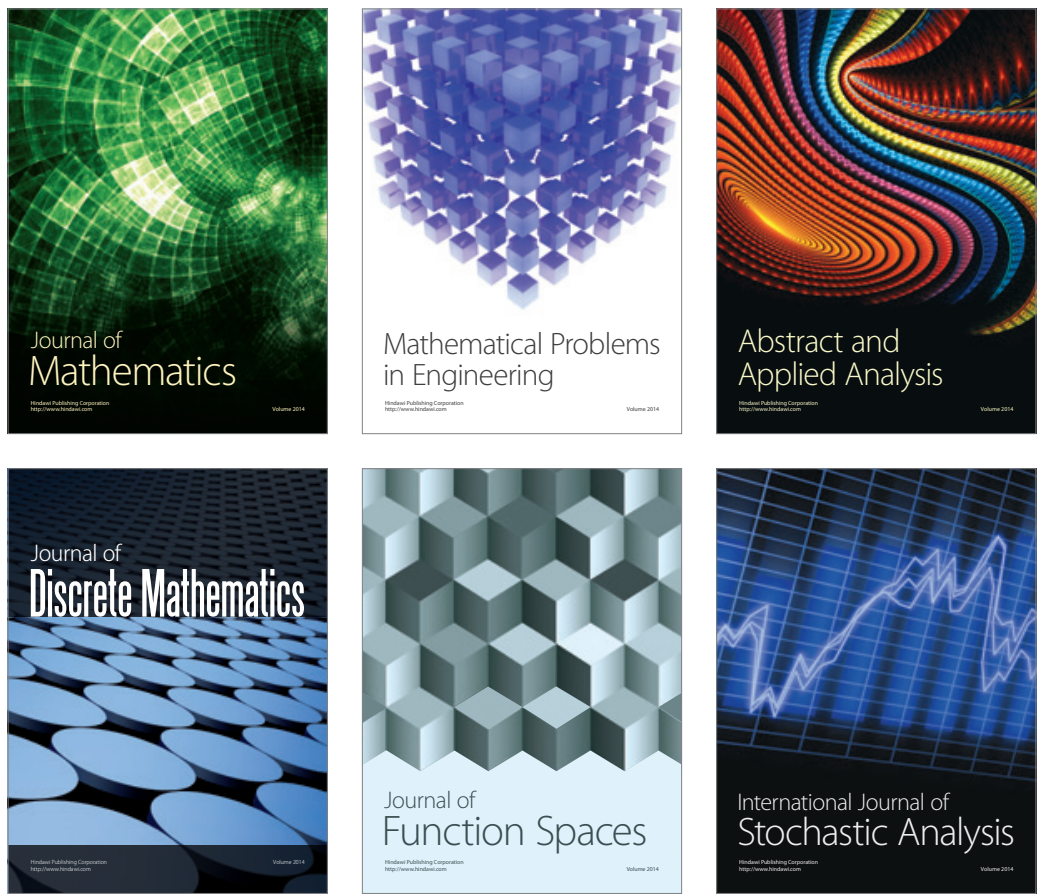

Journal of

Function Spaces

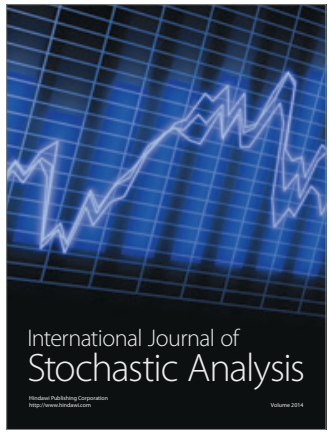

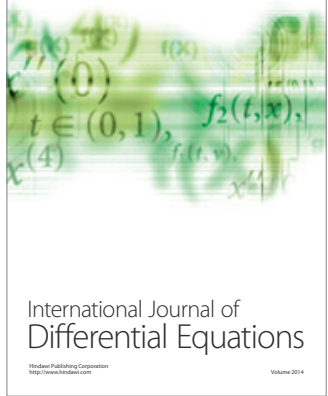
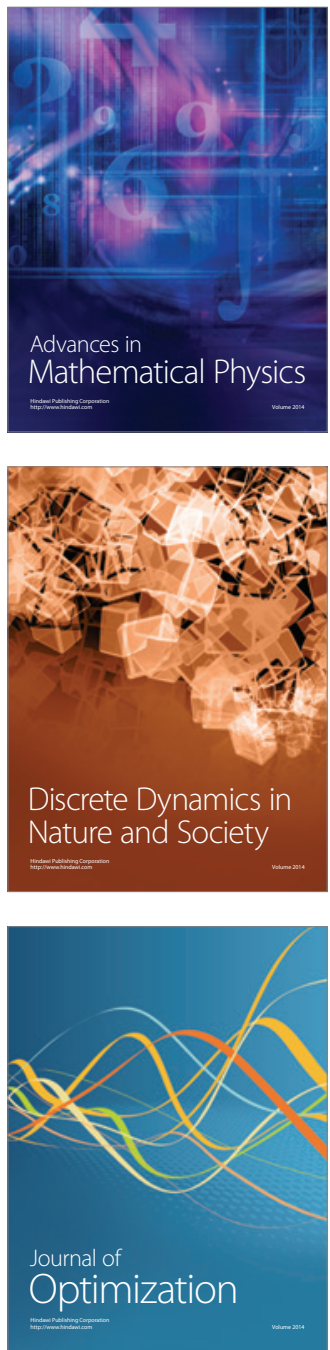